\title{
Semiótica y ciencias humanas: encuentros interdisciplinarios alrededor de las pasiones*
}

Jacques Fontanille

Université de Limoges

Institut Universitaire de France, France jacques.fontanille@unilim.fr

\section{Resumen}

Se presenta en este trabajo una serie de reflexiones correspondientes a la semiótica de las pasiones. Esta última surge de la consideración de dos rasgos característicos de la semiótica que se manifiestan de manera simultánea. El primero está relacionado con el carácter interdisciplinar y el segundo con el carácter indisciplinar.

Se postula la tesis de que la semiótica de las pasiones es un excelente terreno de búsqueda para estudiar las interacciones entre las disciplinas del dominio de las ciencias del hombre y la sociedad. La cuestión de los afectos, de las emociones, de las pasiones no le pertenecen por supuesto en exclusividad a ninguna de ellas, y para constituir su objeto específico, la semiótica de las pasiones ha tenido que componer a la vez con la mayoría de ellas (psicoanálisis y psicología, filosofía e historia de las ideas, ciencias del lenguaje y retórica, antropología y sociología, etc.), y trazar su camino en cuanto dominio y objeto de investigación autónomo.

Palabras clave: Teoría semiótica, pasiones, modelos teóricos, significación, estructura lingüística.

\section{Semiotics and human sciences: interdisciplinary encounters around passion}

\section{Abstract}

It is introduced in this work series of corresponding reflections to the semiotics of the passions. The latter arises from the consideration of two characteristic features of semiotics that manifest simultaneously. The first is related to the interdisciplinary nature and the second to the no discipline feature.

It is postulated the thesis that the semiotics of the passions are an excellent field of research to study the interactions between the disciplines of the domain of the sciences of man and the society. The question about affections, of emotions, of passions does not, of course belong exclusively to any of them, and to constitute its specific object, the semiotics of the passions has had to compose at the same time with the majority of them Psychoanalysis and psychology, philosophy and history of ideas, language sciences and rhetoric, anthropology and sociology, etc.), and to trace their path as the domain and object of autonomous research.

Keywords: Semiotic theory, passions, theoretical models, meaning, linguistic structure.

*Sémiotique des passions, quête du sens et rencontres interdisciplinaires, in Michel Quitout \& El Mosafa Chadli, dir. (2008). La sémiotique: de la narrativité à la mise en discours. Hommage à Georges Maurand, Paris: L'Harmattan. 


\section{Introducción: La semiótica como "interdisciplina"}

La semiótica buscaba, hacia los años 60 y 70 del siglo pasado, convertirse en algo equivalente para las Ciencias del hombre y de la sociedad (CHS), como las matemáticas lo eran para las Ciencias llamadas "exactas". Sin embargo no pudo lograr esta ambición sin duda no solo porque no disponía de los medios científicos e institucionales para llegar a imponerse de esta forma sino también porque las CHS no estaban dispuestas a tener en común un cuerpo de doctrina epistemológica y metodológica. Y sin embargo, de esta ambición insatisfecha y desmesurada, quedó algo importante de lo cual presentaré aquí algunos atisbos. Por un lado, la semiótica se mantiene en una posición 'indisciplinaria'. Y para cada uno de los objetos o de los problemas concretos que aborda, debe dialogar y confrontarse con las disciplinas que en cierta forma son 'locatarias prioritarias' de cada uno de estos dominios de investigación. Por otro lado, buen número de ciencias humanas y sociales, cuando abordan el sentido de los objetos y de las situaciones de las cuales dan cuenta, cuando pasan a su interpretación, hacen semiótica sin saberlo (en un doble sentido). Y esto vale también tanto para la socio-semiótica (que debe confrontar sus resultados con la sociología, llevando a cabo ella misma frecuentes interpretaciones con una tonalidad semiótica) como para la semiótica visual (que debe dialogar con la historia del arte o los especialistas de la información y la comunicación), o la semiótica literaria que debería mantener una estrecha relación con la lingüística textual y con la historia literaria pero que no se da con frecuencia, por diversas razones sobre las cuales no me ocuparé aquí.

Se sabe que la semiótica estructural fundada por Greimas y desarrollada por sus primeros colaboradores se forjó en la intersección de la antropología (Lévi-Strauss), de la folclorística (Propp), de la lingüística saussureana $y$ hjelmsleviana, luego generativa, de la lógica modal, de la fenomenología (Merleau-Ponty) y un poco menos del psicoanálisis (Freud y Lacan).
Sabemos por ejemplo que el principio de la secuencia narrativa canónica se extrapoló a partir de las funciones de Vladimir Propp, y que la tipología de los actantes se inspira a la vez de los roles folclóricos de Propp, de los roles dramáticos de Souriau y de los roles actanciales de la semántica generativa. Sabemos además que la definición de las estructuras elementales y del cuadrado semiótico se encuentra igualmente en la intersección de la lógica filosófica (el cuadrado de Aristóteles), de la fonología estructural (los dos tipos de oposición de Jakobson), y de la teoría del valor en economía y en lingüística (un sistema de relaciones entre diferencias).

Mi objetivo se limitará a la semiótica de las pasiones, nacida a comienzos de los años ochenta, como una prolongación de la semiótica narrativa, en un movimiento teórico y metodológico que comenzó como un simple desarrollo de la teoría de las modalidades y que continuó con un profundo cuestionamiento del principio estructural de oposiciones "discontinuas" entre entidades denominadas "discretas". Esta semiótica de las pasiones se encuentra también en la intersección de la filosofía (la de los sistemas pasionales), de la fenomenología (la del cuerpo propio y del mundo sensible), del psicoanálisis (sobre todo el de las pulsiones, y el de las "barreras psíquicas"), de las ciencias del lenguaje (en particular las teorías enunciativas de la subjetividad), de la retórica (las pasiones persuasivas), de la sociología (las interacciones emocionales) y de la antropología, como se verá al final.

La semiótica de las pasiones es un excelente terreno de búsqueda para estudiar las interacciones entre las disciplinas del dominio de las ciencias del hombre y la sociedad. La cuestión de los afectos, de las emociones, de las pasiones no le pertenecen por supuesto en exclusividad a ninguna de ellas, y para constituir su objeto específico, la semiótica de las pasiones ha tenido que componer a la vez con la mayoría de ellas (psicoanálisis y psicología, filosofía e historia de las ideas, ciencias del lenguaje y retórica, antropología y sociología, etc.), y trazar su camino en cuanto dominio y objeto de investigación autónomo. 
Le debe a la filosofía una muy antigua tradición de tipologías pasionales, los grandes sistemas de las pasiones en Aristóteles, Descartes, Spinoza y Hume entre otros tantos; pero ella se distingue igualmente por una primera toma de decisión teórica propiamente semiótica. Estas tipologías son por supuesto diferentes unas de otras, a pesar de la presión de los legados intelectuales que caracterizan la historia de la filosofía y, en este sentido, dan testimonio de la variación sociolectal e idiolectal propia del universo cultural de cada uno de sus autores. En consecuencia, solo hay una vía propiamente semiótica y generalizable en la organización sintagmática de las secuencias pasionales, y no en la organización paradigmática de las clasificaciones de los nombres de las pasiones propias a cada cultura. En resumen, si la filosofía de las pasiones, de inspiración paradigmática, refleja las diferencias culturales sin darse cuenta, la semiótica de las pasiones, de concepción sintagmática busca un modelo general cuyas variables son por el contrario susceptibles de encargarse de la variación cultural.

Con respecto al psicoanálisis, la deuda es, paradójicamente menor pero en particular indirecta. En efecto, la tentación es grande, al considerar que la semiótica de la afectividad humana ya estaba hecha y que bastaba con recogerla en la semiología freudiana $y$ lacaniana de los afectos; pero las semiologías psicoanalíticas no obedecen necesariamente al principio del empirismo (exhaustividad, adecuación y coherencia), y no buscan además la construcción de la significación de los afectos sino la descripción de la psique. Construida por esta razón, sin referencia explícita al psicoanálisis, la semiótica de las pasiones toma prestado sin embargo (o comparte con él) dos hipótesis fundadoras: (i) su objeto (el afecto, la pasión) no puede ser abordado sin una "encarnación" de los dispositivos teóricos y por lo tanto sin otorgarle al cuerpo sensible un papel en la semiosis; y (ii) los fenómenos de los cuales ella da cuenta, los diversos avatares y manifestaciones de su objeto, expresan las tensiones, fuerzas y presiones que se ejercen sobre el cuerpo de donde emanan. Cada una de estas hipótesis será explotada en dos investigaciones ulteriores: la semiótica del cuerpo y la semiótica tensiva respectivamente.
La semiótica de las pasiones nacida por lo esencial en el seno de las ciencias del lenguaje, se construye sin embargo contra el espíritu dominante del estructuralismo; y es aún una de las características comunes de todas las tentativas propiamente lingüísticas y discursivas de tratamiento de la afectividad como para llegar a disentir en relación con la doxa lingüística. Volveremos más adelante sobre este asunto.

$\mathrm{Su}$ relación con la retórica no es menos asombrosa. A pesar de que la retórica general era el dominio a la vez más próximo de un enfoque semiótico, y el más antiguo interesado en la dimensión afectiva de las interacciones persuasivas, la semiótica ignoró sin embargo el papel de los afectos en las prácticas argumentativas, para consagrarse primordialmente a la "patemización" de las estructuras narrativas. Habrá que esperar a que se interese en las prácticas en general y no solo en los textos-enunciados, para que pueda ser reexaminada la cuestión de los afectos en las interacciones. Volveremos sobre este tema con mayor atención.

Es comprensible que, en estas condiciones, las pasiones colectivas solo hayan sido consideradas tardíamente. Por un lado, las emociones colectivas son para la mayoría, como lo afirma Eric Landowski, "sin nombre" (y sin documento de identificación lexical), $\mathrm{y}$ son en consecuencia fenómenos sociales cuya manifestación solo puede ser totalmente aprehendida por la psicología, el psicoanálisis o las ciencias del lenguaje. Por otro lado, entre más sean colectivas, más caracterizan a grupos numerosos incluso a espacios culturales completos y en menor medida la semiótica del cuerpo y de lo sensible que le proporciona una buena parte de los argumentos de la semiótica de las pasiones es apropiada; siempre es posible invocar una sensibilidad colectiva, des las pasiones y de las tensiones afectivas, pero ellas no pueden ser relacionadas ni con un cuerpo ni con los "movimientos del alma" o con estesias. En última instancia, para un antropólogo que se interesa en los mitos y en el imaginario colectivo, la semiótica de las pasiones es más capaz de dar cuenta de la degradación de un universo colectivo mítico en un universo individual afectivo que de 
circunscribir un dominio de análisis que le sea propio. Volveremos sobre este punto.

Por supuesto, estas intersecciones disciplinarias son demasiado numerosas para ser analizadas en detalle dentro de esta contribución, y se limitará a tres de ellas: (i) las relaciones con las ciencias del lenguaje; (ii) el papel del cuerpotestigo en la persuasión retórica; y finalmente (iii) las relaciones entre la antropología de los mitos y la semiótica de las pasiones.

\section{Afectos, pasiones y estructuras lingüísticas}

Hablar de sentimientos, de afectos, de pasiones y de estados del alma en el campo de las ciencias del lenguaje en el transcurso de los años cincuenta y sesenta constituía una ruptura epistemológica con la doxa estructuralista. Las pasiones solo entraban en el campo de las preocupaciones de las ciencias del lenguaje por efracción y por tres vías diferentes al final de los años ochenta que esbozan poco a poco el campo de la semiótica de las pasiones.

\section{Primera vía: la lingüística de la enunciación}

La primera vía fue abierta por la lingüística de la enunciación: en los años ochenta, la teoría de Benveniste en materia de enunciación y de "subjetividad en el lenguaje", y retomada en especial por Catherine KerbratOrecchionni ${ }^{1}$, permite circunscribir el campo de la modalización enunciativa además de incluir los subjetivemas afectivos ${ }^{2}$. En esta perspectiva, la afectividad parece indisociable de las manifestaciones axiológicas y evaluativas implicadas en los actos de habla. El léxico de una lengua comprende cierto número de lexemas y morfemas cuya significación solo puede ser claramente elucidada se reconstituye, a manera de hipótesis explicativa, la sensibilidad y el juicio de un sujeto de la enunciación; por ejemplo, el adjetivo "triste", solo se interpreta si se le atribuye al juicio de un observador, que aprecia el contenido de un enunciado en referencia a su propia escala de sensibilidad.

Pero como es, este análisis se basa únicamente en dos actos cognitivos no necesariamente pasionales: por un lado, una evaluación situada en una rejilla de referencia (es el papel del observador) y por otro, un predicado de juicio (es el papel del enunciador propiamente dicho). "Triste" remite sin duda a una capacidad para emocionarse, pero que permanece virtual $y$ no está expresada aquí en cuanto emoción. Podemos entonces hablar de juicio afectivo, incluso de escala de sensibilidad, pero no de manifestación emocional; el enunciado no manifiesta ni la emoción ni el afecto sentidos, sino únicamente un valor subjetivo que resulta de un juicio estandarizado. La "subjetividad lingüística" no da pues acceso a la afectividad propiamente dicha.

\section{Segunda vía: la fenomenología del amor}

La segunda vía fue abierta por el análisis del discurso amoroso hecho por Barthes: Fragmentos de un discurso amoroso ${ }^{3}$ se pierde sin dificultad $\mathrm{y}$ voluntariamente en la subjetividad afectiva: discurso en primera persona, sin metalenguaje, él reconstituye y comenta escenas y motivos ${ }^{4}$ típicos del amor, vividos desde el interior: el análisis del discurso amoroso se transforma en fenomenología de lo vivido como enamorado:

Se ha sustituido pues la descripción del discurso amoroso por su simulación, y se le ha restituido a este discurso su persona fundamental, que es el yo, de manera de poner en escena una enunciación, no un análisis. Es un retrato, si se quiere, lo aquí propuesto; pero este retrato no es psicológico, es estructural: da

1 Catherine Kerbrat-Orecchionni, L'énonciation. De la subjectivité dans le langage, Paris, Armand Colin, 1980, rééd. 1997.

2 Op. cit., pp. 70-120.

3 Roland Barthes, Fragments d'un discours amoureux, Paris, Seuil, 1977. [Fragmentos de un discurso amoroso, traducción española de Eduardo Molina, México, Siglo XXI editores, 1982]

4 Son los propios términos de Barthes, en particular el acápite Cómo está hecho este libro, pp. 13-18. 
a leer un lugar de palabra: el lugar de alguien que habla en sí mismo, amorosamente, frente a otro (el objeto amado) que no habla ${ }^{5}$.

Este enfoque es fenomenológico en el sentido en que se esfuerza en hallar el núcleo sensible de la experiencia amorosa, y lo probado en cada una de sus situaciones o figuras. Pero, al mismo tiempo, ya no se tiene acceso a la significación objetivable de estos afectos. La dificultad puede resumirse así: ¿Cómo describir la emoción y la afectividad enunciadas sin apagar la manifestación enunciativa y expresiva? ¿Cómo tomar la vibración de la emoción o de la pasión sin morder el anzuelo del juicio, de la evaluación cognitiva y de las rejillas culturales?

\section{Tercera vía: la semántica modal y la "masa fórica"}

La tercera pista se abrió en el seno mismo de la semiótica narrativa a través de la teoría de las modalidades. En 1979, aparece el primer artículo de Greimas que le da vía libre a una semiótica de las pasiones con un fundamento modal: «De la modalisation de l'être» ${ }^{6}$. Después de haber consagrado los años que preceden a la competencia modal del sujeto narrativo, Greimas aborda la existencia modal, es decir, las modalidades del ser (deber-ser, querer-ser, poder-ser, etc.).

Pero propone también una solución para resolver la dualidad problemática de los enunciados pasionales: Greimas introduce en efecto una categoría nueva, la "foria" (literalmente; lo que conduce a...), y explica cómo engendra la categoría de la modalización. Las dos categorías manifiestan los mismos fenómenos afectivos, pero de forma diferente, una como fuerzas indistintas y movimientos de atracción y de repulsión, en resumen como emociones elementales, y la otra, en una serie de contenidos modales interdefinidos y objetivables. Es así que la foria (euforia y disforia de los humores $\mathrm{y}$ emociones elementales), se transforma en una distribución de modalidades: deseable/ indeseable (según el querer), indispensable / dañino (según el deber), posible / imposible (según el poder), etc..., que describen estados afectivos más específicos.

En este sentido, la semiótica intenta resolver el aporte metodológico de las ciencias del lenguaje que solo podrían dar cuenta de la emoción colocándola entre paréntesis, o solo podían restituirla privándose de toda descripción explícita. Y es esta solución que ha hecho posible el despliegue de una verdadera semiótica de las pasiones que asocia siempre sin debilitar por un lado la explicitación y la categorización de los contenidos pasionales y por el otro, la gestión de las tensiones y movimientos corporales y sensibles.

\section{Ethos, pathos: el corpus persuasivo}

El cuerpo persuade tanto como el verbo. Persuade sobre todo en el ejercicio de la palabra, lo que la retórica ya identificó e integró a la acción. En consecuencia, la construcción de la instancia persuasiva de toda enunciación debe tener en cuenta las figuras del cuerpo. Y la primera consecuencia de esta opción teórica corresponde a la articulación entre el ethos y el pathos y entre la argumentación y la emoción.

El ethos corresponde a la vez a la imagen de sí previamente admitida por los compañeros y a la presentación de sí en el acto de discurso, y ambas dependen de las manifestaciones pasionales y emocionales. Ciertamenteel pathos esconsiderado en general desde el punto de vista de los efectos producidos en el enunciatario, pero estos efectos no solo se derivan del contenido cognitivo de la argumentación, ya que, teniendo en cuenta el principio de contagio y de participación enfática que gobierna los intercambios pasionales, resultan también pasiones y emociones expresadas por el enunciador. Desde esta perspectiva, las estrategias argumentativas pueden ser consideradas también como el soporte del contagio pasional.

5 Op. cit., p. 13 de la versión española.

6 In Actes Sémiotiques, Bulletin, 9, pp. 9-19. Retomado en Du sens II, Paris, Seuil, 1983, pp. 93-102. 
Por lo tanto, la argumentación puede ser concebida como un intercambio de "simulacros" pasionales que acompañan a las representaciones que cada uno de los participantes se hace de sí mismo y de los otros. El ethos del enunciador comprende estados pasionales y emociones recurrentes que entran en el simulacro de su "carácter" o de su "temperamento"; y el pathos del enunciatario solo puede ser modificado por el enunciador si este último dispone de un simulacro modal y patémico de su compañero. En resumen, el ethos integra una parte del pathos, y el pathos comprende una parte del ethos.

Además la participación del cuerpo, carne o cuerpo propio, modifica las propiedades del intercambio argumentativo, ya que modifica la naturaleza misma del "lazo" persuasivo entre los participantes.

\section{El cuerpo como prueba patética}

Para Aristóteles, el ethos hace parte de las pruebas retóricas, con respecto a las costumbres del orador ${ }^{7}$. Entre estas pruebas constitutivas del ethos, algunas son producidas por el cuerpo enunciativo mismo: Las manifestaciones pasionales del orador modifican las del oyente sobre todo a través de las manifestaciones concretas y las propiedades del cuerpo enunciante. El ethos del orador, por lo tanto no está constituido solamente por su competencia, por sus atributos y por sus antecedentes (narrativos), sino también por su "temperamento" (pasional y emocional) anclado y manifestado en un cuerpo ${ }^{8}$.

En este sentido, el ethos encarnado (el cuerpo ético-patético) es una condición de "acreditación" del enunciado: inspira confianza en el sentido en que el proceso de contagio pasional, la respuesta afectiva de uno es una forma de "crédito" concedido a la solicitud/proposición por parte del otro. El cuerpo ético-patético le ofrece entonces "crédito" a la enunciación, crédito que se refuerza con la copresencia de cuerpos vivos y sensibles que comparten las mismas experiencias y las mismas emociones y que sostiene a su vez la fuerza persuasiva de los discursos manifestados.

\section{La relación entre el acto y la persona}

Para Chaim Perelman, la eficacia del ethos se basa (desde es un punto de vista filosófico y ético) en el principio de la relación entre el acto y la persona que lo realiza ${ }^{9}$. El ethos del orador puede vincularse con la persuasión si su enunciado si solo es considerado no como universal e impersonal, sino como el producto de una enunciación personal, por un acto atribuible a un individuo y que apenas absorbe una parte de su valor en la identidad de este individuo. A esto se le puede añadir que la "unión" en cuestión tiene como fundamento principal el carácter corporal de la enunciación.

Perelman considera dos grandes tipos de estrategias persuasivas: las que asumen la relación entre acto y persona (procedimientos de asociación y de refuerzo), y las que no lo asumen (procedimientos de disociación y de frenado en la relación entre el orador y sus argumentos). En este sentido, las manifestaciones afectivas y sus expresiones corporales puedan ir en dos direcciones: una expresión facial de escepticismo, que acompaña a un argumento basta para "frenar" la relación entre acto y persona $\mathrm{y}$

7 Aristote, Rhétorique, LI, 2, 1356a-1. [Retórica, traducción española de Alberto Bernabé, Madrid, Alianza editorial, 2004]

8 En las concepciones de la argumentación, el cuerpo sensible recupera todos los derechos: por ejemplo, para Dominique Maingueneau (Le contexte de l'cuvre littéraire, Paris, Dunod, 1993), el ethos proviene de la instancia de enunciación siempre que esta última sea más formal y se reduzca a un papel actancial pero al tiempo que sea también una 'voz', un ritmo, una carne, un cuerpo: de manera global se trata entonces de "propiedades que los hablantes se atribuyen a través de su forma de hablar" (1993:137).

9 Chaïm Perelman, Traité de l'argumentation. La nouvelle rhétorique, Bruxelles, Editions de l'Université Libre de Bruxelles, 1988. [Tratado de la argumentación. La nueva retórica, versión española de Julio Sevilla Muñoz, Madrid, Gredos, 2006.] 
para proteger el ethos del orador de los efectos de la retroalimentación de una proposición cuestionable; por el contrario, un tono de voz cálido y entusiasta, puede convertir un argumento débil en una proposición totalmente asumida.

En resumen, el compromiso y la liberación física y emocional igualmente pueden debilitar o fortalecer el vínculo entre el acto y la persona, y la atribución de la primera en la segunda.

Ya que para enunciar legítimamente y de manera creíble, el orador debe de alguna forma dar testimonio de una experiencia personal: un orador debe tener un cuerpo, un cuerpotestigo que, habiendo vivido lo que argumenta, conserva los rastros y las huellas: ya sea sincero o afectado, el acompañamiento somático-afectivo de la argumentación se deriva de la puesta en escena de un "testimonio".

Mitos y pasiones; diálogo de frontera con la antropología

\section{Problemática}

El discurso mítico manipula configuraciones cosmológicas, narrativas y prácticas que en principio nada tienen de pasional. Pero sucede que para la conversión de estas configuraciones en otros géneros culturales, incluyendo los literarios, deben convertirse explícitamente en pasionales. Y sucede incluso que esta conversión pueda tener lugar al interior mismo del texto mítico. El mito sufre entonces transformaciones que pueden hacerlo desconocido; y por el contrario, la pasión "mitificada" ya no dispone de ninguna propiedad afectiva.

\section{La cólera de los dioses y la cólera de los hombres}

Los mitos y leyendas ofrecen un muy amplio abanico de variedades no afectivas de la cólera: son ejemplos de ello, en el ámbito indoeuropeo la cólera de Cuchulainn ${ }^{10}$ o la de Manyu ${ }^{11}$. En este caso, la cólera es tratada como una variante de la violencia esencial y de la energía cósmica, y se acerca en sus relaciones con lo sagrado, con el orden del mundo y de la sociedad. Pero la ira divina no es calamitosa: solo es miedo. Por el contrario, la ira de los héroes humanos o semidivinos, incluso si no es afectiva, debe ser contenida y reprimida ya que es contagiosa para los hombres con los cuales tiene relación.

La intensidad, la cantidad y la mediación $\underline{\text { mítica }}$

En los mitos y las cosmogonías indoeuropeas, la ira no es más que una variante de la energía, y ella emana directamente del ser; y la transformación crucial, la que pasa del ser a la existencia, solo se realiza bajo el precio de una disminución de esta energía y de una entrada en la extensión y la cantidad. Solo los dioses poseen su propia cólera-energía y, en el panteón indio, particularmente Manyu. En efecto, en los himnos religiosos más antiguos, el dios Courroux, Manyu tiene el estatus de "fuerza primordial"12; es la fuerza misma del ser, la que le permite arrancar el ser del no-ser y de hacer existir el cosmos.

La ira divina se manifiesta sin agotarse; Manyu siempre está furioso. Este es un fenómeno que Séneca no hubiera podido explicar, aquel para quien la explosión de ira solo conduce al debilitamiento. En este caso, como se ve, ninguna confianza traicionada, ninguna decepción, la ira divina o cuasi divina no tiene necesidad de justificación; ella es su propia razón, y en lugar de debilitarse al manifestarse, esta ira parece estar destinada a durar por siempre. El discurso mítico, ubica pues la ira directamente en la experiencia de la "resistencia" de los estados de cosas, en la tensión entre el ser y la existencia,

10 En La geste de Cuchulainn, G. Roth, Alfortville, L'Edition d'Art, 1927.

11 Cf. C. Malamoud, "La colère et le désir dans le système des passions royales de l'Inde ancienne", Psychiatrie française, La colère, 1992, 1, pp. 8-14.

12 C. Malamoud define de la siguiente manera el dios sánscrito Courroux: Manyu es también una fuerza nacida de sí misma, sin origen, pero ella misma es origen de todo, la primera intención, pasión germinal contra el no ser (op. cit., p. 10). 
exactamente en donde Heidegger construirá la configuración de la "Preocupación" por el Dasein. Esta ira existencial no posee nada afectivo sino que tiene relación con el orden del mundo y su estabilidad.

La intensidad o la extensión: la alternativa de la ira humana

Este imaginario mítico de la ira deja huellas en las representaciones más tardías y más banales. En el De Ira, por ejemplo, después de haber denunciado la ineficacia de la ira, Séneca defiende el aplazamiento, la demora, pero para terminar añade un curioso consejo:

El mejor remedio para la ira es el tiempo. No le pidas al principio que perdone, sino que juzgue; si espera, se disipa. No trates de comprimirla de un solo golpe; su primer arrebato es demasiado enérgico; pero se la vence por completo si se la ataca por partes ${ }^{13}$.

Una vez que ya no se trata de la ira divina, la intensidad y la cantidad pasionales se combaten entre sí; el mínimo estiramiento temporal disminuye la intensidad de la ira y hace de ella un resentimiento difuso, o un proyecto de venganza calculada; el mínimo análisis en partes o en aspectos conduce al mismo resultado: la extensión y la cantidad combaten la intensidad: El sujeto humano con ira tiene un problema que resolver que es típicamente pasional y que no tiene nada de mítico: se trata de permanecer ya sea "completo y único" en la fuerza de la pasión inmediata, ya sea convertirse en algo "múltiple y diverso" en la relación con el otro y con el mundo, en detrimento de la fuerza de la manifestación pasional.

\section{La ira entrópica}

La ira humana reposa en una tensión inversa entre la fuerza de explosión y la extensión (la cantidad de represalias, la duración del resentimiento, etc.) mientras que la ira divina y mítica no opone estas dimensiones: entre más grande y amplia sea la manifestación, más intensa permanecerá.

Pero se pueden hallar situaciones intermedias, en donde ellas se confunden de manera aterradora: la ira de los héroes legendarios y de los reyes, mitad hombres, mitad dioses es entrópica. En la India antigua, vemos que junto a los himnos religiosos que magnifican el Courroux divino, el que suscita y mantiene el ser, otros textos hacen de la ira el peor de los pecados: Kautilya por ejemplo, en su tratado político Artha-shastra ${ }^{14}$ considera la ira como la "primera pasión calamitosa" para los reyes, y justifica este rango por su poder de diseminación; él nos explica que la ira del rey engendra la de los adversarios, la de los vecinos, la de todo el pueblo, etc. La violencia existencial (la ira-energía) es creadora para los dioses, pero destructiva para los hombres que juegan a ser dioses, ya que amenazan la existencia misma de su comunidad. En consecuencia, hay que mantener y contener cada tipo de ira en su propio dominio.

Los hombres inventaron pues como solución a su medida, una simple pasión que no tiene que ver con la violencia ontológica y cósmica que es prerrogativa de los dioses y los héroes míticos: la ira humana tiene sus razones, ella ocurre como reacción a una ruptura unilateral de una relación de confianza, se agota cuando se expresa y se desarrolla en una secuencia canónica entre en comienzo y un fin, una secuencia que controla la tensión entre la intensidad y a cantidad que las hace solidarias pero según una solidaridad inversa, que nos obliga a escoger entre la violencia sin mañana y unas monedas de resentimiento por un largo tiempo.

13 De la ira, Lucio Anneo Séneca; traducción directa del latín por Francisco Navarro y Calvo. Alicante : Biblioteca Virtual Miguel de Cervantes, 1999

14 Kangle, R.P., The Kautilîya Arthaçâstra, Part 1, A Critical Edition with a Glossary, Bombay 1960, University of Bombay, Part 2, An English Translation with Critical and Explanatory Notes, Bombay 1963, University of Bombay, Part 3, A Study, Bombay 1965, University of Bombay. 
Una es la fuerza cosmológica sin desarrollo sintagmático y sin historia; la otra es una pasión, reconocible por su forma canónica en donde se conjugan, reforzándose la intensidad vital y la extensión existencial mientras que la cólera humana pone en escena una resolución sintagmática de una tensión inversa entre las dos dimensiones.

\section{Los celos, entre semiótica y antropología}

El segundo caso es el de los celos para los cuales existe ya un notable texto antropológico que pone en escena los dos estados de la configuración, el estado "mítico" y el estado "pasional" y que sugiere sus posibles articulaciones. Se trata de La Alfarera celosa ${ }^{15}$ de Lévi-Strauss: la alfarería y los celos son explícitamente puestos en relación y quisiéramos mostrar que ellos se presentan como dos formas alternativas de resolver la misma tensión-contradicción.

\section{La alfarería, la guerra y los excrementos}

Lévi-Strauss estudia una familia de mitos amerindios que combinan y alternan los celos y la alfarería. Para comenzar, debemos recordar que el análisis semiótico de los celos, tal y como fue propuesto en la Sémiotique des passions ${ }^{16}$, localiza la tensión afectiva principal en la imposible y dolorosa superposición de dos estructuras actanciales, cada una descansando en la relación entre dos roles narrativos: por un lado, la rivalidad entre sujetos y por el otro, la unión entre sujeto y objeto. Estas dos relaciones son solidarias e incompatibles al tiempo: la unión con el objeto exacerba y perturba la rivalidad entre sujetos; la rivalidad intensifica y compromete la unión con el objeto. El afecto suscita al tiempo al tiempo tanto la solidaridad y la incompatibilidad entre las dos configuraciones actanciales: la mediación, sin embargo necesaria se ha vuelto imposible.
La alfarera celosa de Lévi-Strauss describe y comenta por su lado los desdoblamientos y transformaciones de varias temáticas narrativas y figurativas: la alfarería (su invención; su uso; su material de base, la arcilla); los celos (entre parejas, entre dioses, entre dioses y hombres); la guerra (la separación de los clanes, su hostilidad, sus combates, la discordia en general, entre el cielo y la tierra, entre los hombres, etc.); la retención y la incontinencia corporales (el estreñimiento y la defecación, la modestia y la falta de modestia; la abstinencia y la bulimia sexuales, la avidez o la retención oral, verbal, material o simbólica).

La alfarería se relaciona con los celos en cierta forma por deformación profesional: el alfarero supervisa de manera exclusiva e intransigente la calidad, la regularidad y la pureza del conjunto del proceso de fabricación y sus "celos" protegen de alguna manera la alfarería de toda intervención exterior intempestiva.

La alfarería es un poderoso factor de síntesis para las demás temáticas: del lado de la guerra y de los conflicto, ella recibe su "materia prima": es después de un conflicto que uno de los combatientes, humano o divino, relajándose, produce la arcilla con la cual se harán vasijas. La arcilla de las vasijas está presente además en los dos extremos de la cadena de la continencia/ incontinencia corporal: a la entrada, como "alimento" y a la salida, como "excremento". Cabe recordar aquí que estas dos temáticas, la guerra y la continencia/incontinencia corporales, le hacen eco a los dos ejes principales de pasión y de los celos: por un lado, la rivalidad, y por el otro, el apego exclusivo (retención). La alfarería los asocia, neutralizando toda contradicción entre ellos, e incluso los encajona de manera irreversible el uno en el otro.

Los celos recubren en estos mitos toda una gama pasional, la envidia, los celos, la avidez y la avaricia ${ }^{17}$. Nos encontramos por ejemplo

15 Claude Lévi-Strauss, La potière jalouse, Paris, Plon, 1985. [La alfarera celosa, traducción española de Caterina Molina, Barcelona, Paidós, 1991.]

16 A. J. Greimas et J. Fontanille, Sémiotique des passions, 1991, op. cit., tercera parte. [Semiótica de las pasiones, traducción española de Gabriel Hernández Aguilar y Roberto Aguilar, México, Siglo XXI Editores, 1994].

17 Lévi-Strauss, op. cit., p.109. 
verdaderos celosos, maridos y mujeres, pero tanto hombres como mujeres que se apoderan del otro ya sea por fraude o por la fuerza; encontramos dioses que no aceptan ninguna indiscreción por parte de sus seguidores, o de los artesanos (cf. supra) que protegen sus fabricaciones de toda intrusión indeseable. Se trata pues globalmente de una gama de comportamientos que no contienen necesariamente un componente afectivo sino solamente, en cuanto clase narrativa, una dimensión de retención (exclusiva) o de apropiación ávida y agresiva (excesiva), es decir, precisamente, de nuevo, los dos tipos de situación narrativa que constituyen los celos "afectivos" (el apego al objeto y el conflicto con el rival).

La alternativa: mediación paradigmática $\mathrm{O}$ resolución sintagmática

Los mitos se inscriben en dos temáticas narrativas, las mismas que se obtuvieron por el análisis de los celos "afectivos": (i) por un lado. los conflictos, combates, y problemas cósmicos o de clanes; y (ii) por el otro, la avidez y la retención que describen la relación con los objetos de deseo y apego. Estas dos configuraciones son solidarias e incompatibles y el mito intenta resolver esta superposición problemática. Pero los relatos míticos consideran dos soluciones diferentes: una mediación paradigmática y figurativa, o una resolución sintagmática y pasional.

En el primer caso, el tratamiento de la tensión identificada no es afectivo sino figurativo. En la intersección de la rivalidad y del apego, puede entonces aparecer cualquier cosa menos un despliegue apasionado: de la alfarería, un oficio, una clase de objetos característicos de la actividad cultural. En lugar de la afectividad, que nace de una tensión insoluble entre dos cuestiones, el pensamiento mítico, como Lévi-Strauss y otros lo establecieron, busca una mediación figurativa.

En este sentido, la alfarería propone una "mediación semántica" (y paradigmática), mientras que la afectividad propone una gestión secuencial (y sintagmática). En cuanto actividad de producción cultural, la alfarería constituye una resolución por mediación; se ha visto en efecto cómo la alfarería explota los productos materiales de los conflictos y participa por lo tanto de los motivos de la retención, de la incontinencia y de la continencia corporales. Los celos, por el contrario, son una resolución por secuencia: gracias al despliegue sintagmático de una tensión insoluble, solo puede encontrar su sentido, precisamente ligándose con la forma de una secuencia canónica.

$Y$ es por lo que Lévi-Strauss señala en repetidas ocasiones que tanto el motivo afectivo y conyugal es como una "miniatura" de los grandes conflictos cósmicos que se encuentra en otros mitos de las mismas culturas ${ }^{18}$ : una miniatura, sin duda, ipero después de la conversión semiótica!

\section{Conclusión}

La semiótica es una interdisciplina que desarrolla un papel sucesivo reconfigurando la substancia del contenido propuesto por las demás disciplinas, a fin de elaborar la esquematización común, a posteriori. Este procedimiento es la forma misma de la búsqueda del sentido, ya que buscando resolver la heterogeneidad de estas substancias de contenido multi-disciplinares, construye poco a poco la significación, que es, transdisciplinaria.

Ella interviene después ya que tiene necesidad de todo el trabajo acumulado por otras disciplinas, datos que ellas han elaborado, primeras interpretaciones que verificaron, $\mathrm{e}$ hipótesis explicativas que formularon. Es raro que el enfoque semiótico haya introducido, en los dominios que ha explorado, hipótesis totalmente nuevas, cuyos especialistas del dominio no tenían la misma intuición. Se cita con frecuencia la evolución considerable de los estudios arquitectónicos bajo la influencia de la semiótica, sobre todo la puesta en evidencia de las interacciones narrativas, modales $y$ pasionales entre lo construido y los actores y

18 Op. cit., p. 59. 
los colectivos. Pero un estudio historiográfico preciso mostraría sin ninguna duda que esta evolución ya estaba en germen, o incluso formulada por los investigadores en arquitectura $y$ que encontraron en el enfoque semiótico el método, los conceptos y los argumentos para sustentar su propia posición. Podríamos plantear la misma pregunta a propósito de la "invención" de la dimensión plástica en las obras visuales: tal parece que remite a la semiótica visual, y a las primeras distinciones en Greimas y en Floch entre lo figurativo y lo plástico. Pero no se excluye, en este caso, que un estudio historiográfico más preciso pueda mostrar que esta distinción estuviera presente en las formulaciones teóricas de algunas corrientes estéticas, si no en la obra de pintores contemporáneos.

En resumen, ella conduce datos multidisciplinarios hacia una significación transdisciplinaria, según un proceso que amerita, para terminar, se evocado rápidamente. El primer gesto, es el de la constitución de los datos, que es la primera etapa del trabajo científico. Pero si el objetivo es la construcción de la significación, y no simplemente el respeto de las reglas de método de una disciplina dada, la naturaleza misma de los datos es por principio heterogénea y abierta provisionalmente. Y como no se puede esperar el fin del proceso de análisis para decidir cuáles son el perímetro y la composición pertinentes para la recopilación de los datos, con el fin de obtener una significación óptima, la construcción de la significación y la de los datos son llevadas en conjunto, progresivamente por acumulación y densidad progresiva del análisis. El paso de lo "multidisciplinar" a lo "transdisciplinar" no se hace pues, desde un punto de vista semiótico no por una inclinación de un campo hacia otro, puntos de vista múltiples de las disciplinas involucradas hacia el punto de vista único de la semiótica, ni por un tipo de toma de poder y de distancia, radical y discontinua, que señalaría la entrada en el dominio semiótico, aquel en donde se accede por fin al sentido. Este paso es por el contrario progresivo, continuo, y podríamos incluso decir que es justamente el rol de la semiótica el que asegura esta progresión y esta continuidad en la transición entre la contribución de varias disciplinas distintas y la construcción de un sentido transversal y común a todas ellas.

\author{
Versión española de \\ Miguel Ángel Mahecha B., \\ Universidad Surcolombiana, Colombia
}

